\title{
ANÁLISIS AL NUEVO SISTEMA DE ADMISIÓN ESCOLAR EN CHILE: LA REGIÓN DE MAGALLANES COMO EXPERIENCIA PILOTO
}

\author{
Mario Sillard ${ }^{1}$ \\ Mario Garay ${ }^{2}$ \\ Ivka Troncoso ${ }^{3}$
}

\begin{abstract}
RESUMEN
La segregación socioeconómica escolar es un fenómeno que ha venido despertando un creciente interés en el país. La entrada en vigencia de la Ley de Inclusión Escolar en el año 2016 convirtió al proceso de elección de escuela en un asunto relevante dentro del debate de las políticas educacionales, al poner fin a la selección de alumnos por parte de los establecimientos que reciben subvención del Estado. La finalidad de la normativa es impedir cualquier tipo de discriminación, lo que en la práctica implica que, si los establecimientos elegidos por las familias cuentan con igual número de postulantes y vacantes, todos los estudiantes podrán ingresar automáticamente. El objetivo de este estudio fue comprender cómo se comporta la matrícula con el Nuevo Sistema de Admisión (NSA) que se implementó como plan piloto en la Región de Magallanes, Chile, para el proceso 2017. En función de ello, se revisaron los datos proporcionados por el Ministerio de Educación (Mineduc), buscando responder a la pregunta de si el NSA permite una distribución de la matrícula de los estudiantes diferente de la del sistema antiguo.
\end{abstract}

Conceptos clave: cuasimercados educativos, desigualdad educativa, elección escolar, segregación escolar.

\section{ANALYSIS OF THE NEW SCHOOL ADMISSION SYSTEM IN CHILE: THE REGION OF MAGALLANES AS A PILOT EXPERIENCE}

\section{ABSTRACT}

The phenomenon of the socioeconomic segregation of schools has surfaced as a prominent concern in the country. The School Inclusion Law came into effect, in 2016, transforming the school selection process into a relevant issue within the educational policy debates by prohibiting schools, that receive state funding, from selecting students. The goal of this legislation is to prevent discrimination, such that if the school chosen by the family has the same number of applicants and vacancies, all of the students should be able to enter automatically. The objective of this

1 Departamento de Educación, Universidad de Magallanes, UMAG, Punta Arenas, Chile. Contacto: mario.sillard@umag.cl

2 Departamento de Educación, Universidad de Magallanes, UMAG, Punta Arenas, Chile. Contacto: mario.garay@umag.cl

3 Departamento de Educación, Universidad de Magallanes, UMAG, Punta Arenas, Chile. Contacto: ivka.troncoso@umag.cl 
research is to understand how school registration behaves with the New Admission System (NAS), which was implemented as a pilot programme in the region of Magallanes, Chile, for the 2017 admission's process. On this basis, the data provided by the Ministry of Education (Mineduc) was reviewed, to answer whether, in comparison to the previous system, the NSA allows for a different distribution of the students' registration.

Keyconcepts: educational quasi-markets, educational inequality, school choice, school segregation

\section{Presentación}

El sistema educacional chileno es el resultado de las dinámicas de mercado socioeducativo que se han llevado a cabo por más de tres décadas. En este caso particular, no se puede negar la existencia de múltiples canales de desigualdad, donde la estratificación socioeconómica pareciera que representa una de las formas de segregación más relevante, si se la compara con las raciales, étnicas o de género (Valenzuela, Bellei y Ríos, 2010). Esto se debe, por un lado, al aumento de la cobertura de las escuelas particulares subvencionadas y, por otro, al desprestigio de la educación pública, el sistema de copago y la selección de los alumnos que hacen las escuelas (Bellei 2009; Elacqua, 2012; Valenzuela, Bellei y Ríos, 2014). Con estos elementos imperantes en el sistema educativo, se hace complejo pensar en una escuela pública, gratuita, de calidad y socialmente integrada: este anhelo educativo aparece como un imposible en el imaginario de amplios sectores de la sociedad chilena actual (Canales, Bellei y Orellana, 2016).

Aunque la selección de alumnos por motivos socioeconómicos fue prohibida por la Ley 20.370 General de Educación de 2009, queda de manifiesto que determinadas prácticas, enquistadas en la tradición, producen segregación escolar y privilegian los mecanismos de cuasimercado y la libertad de elección de las familias, generando una inequidad en el sistema educativo. Dicho de otro modo, la segregación se produce cuando los hijos de familias de menor nivel socioeconómico asisten a establecimientos municipales, mientras que las familias con mayores ingresos y estudios se concentran en el sector particular subvencionado o particular. Esto se refuerza con las prácticas de selección de estudiantes por parte de las escuelas, especialmente en 
las privadas (Contreras, Bustos \& Sepúlveda, 2007), ya que diversos estudios han demostrado cómo la selección continúa siendo una práctica habitual en muchas de ellas (Carrasco, Bogolasky, Flores, Gutiérrez y San Martín, 2014; Contreras et al., 2010). En resumen, la educación chilena se ha segmentado socialmente, de modo que el perfil socioeconómico está muy marcado por el tipo de establecimiento al que asisten los estudiantes: las escuelas privadas atienden a familias de nivel socioeconómico alto; las escuelas privadas subvencionadas — que reciben recursos del Estado y pueden cobrar un arancel obligatorio, sin perder el subsidio—, albergan a familias de nivel medio; y las escuelas municipales atienden principalmente a familias de nivel socioeconómico medio-bajo y bajo (Valenzuela et al., 2014).

\section{Antecedentes teóricos}

La Ley 20.845 de Inclusión Escolar de 2016 pretende regular el mercado educativo y establecer parámetros éticos mínimos para el funcionamiento de las escuelas que reciben recursos públicos, con lo que se espera favorecer la integración social en el sistema educativo y terminar así con la segregación que ha sido la tónica en estos últimos años.

Dicha ley explicita y norma respecto de temas de diversidad, integración, exclusión y convivencia. Aborda la diversidad desde una expresión más amplia, relevando el hecho de que esta no constituye una amenaza, sino que por el contrario educa y enriquece a la comunidad escolar y a la sociedad. Así mismo, la legislación incorpora la noción de igualdad de oportunidades cuando señala que es "deber del Estado velar por la igualdad de oportunidades y la inclusión educativa, promoviendo especialmente que se reduzcan las desigualdades derivadas de circunstancias económicas, sociales, étnicas, de género o territoriales, entre otras" (Ley 20.845, artículo 4). Con ello se busca asegurar que las niñas y niños accedan desde sus primeros años de vida a una educación equitativa y de calidad, considerando la inclusión escolar como el espacio de integración de clases sociales, bajo la premisa de que al incluir socialmente a los estudiantes se pueden generar ambientes sociales más cohesionados (Rojas, Falabella y Alarcón, 2016). En este contexto, la ley explicita que las escuelas ya no pueden tener barreras de selección e identifica 
su calidad educativa con hacerse cargo de los diferentes perfiles de los estudiantes, lo que debería generar las condiciones para un sistema escolar con mayor integración sociocultural. Es decir, cuestiona los hallazgos de investigaciones que indican que los mejores resultados de los establecimientos se explican, principalmente, porque estos seleccionan el perfil socioeconómico de los estudiantes y no necesariamente debido a la efectividad de sus procesos formativos (Carrasco et al., 2014).

En consistencia con lo anterior, el sistema de admisión que se está implementando en modalidad de pilotaje en la Región de Magallanes tiene como finalidad mejorar el antiguo sistema, en primer lugar para facilitar el proceso de postulación y ampliar la oferta de colegios para los alumnos y, en segundo término, para resguardar que el proceso sea objetivo, transparente e iguale las oportunidades a padres y apoderados que postulan a sus hijos a establecimientos municipales y particulares subvencionados.

En este sentido, un efecto que se pudo constatar durante las postulaciones para el ingreso 2017 fue que muchos establecimientos tenían más postulantes que cupos disponibles. Así, para salvar la brecha entre la oferta y la demanda, los postulantes se ordenan en una lista que respeta las prioridades indicadas en la Ley de Inclusión (vínculos familiares, vulnerabilidad económica, parentesco con funcionarios y reintegración), además de garantizar la igualdad de oportunidades en caso de empates — que se resuelven de manera aleatoria-, declarando explícitamente que la selección no está dada por resultados de pruebas o que la exclusión se basa en aspectos socioeconómicos o culturales de los niños o sus padres.

En resumen, este sistema tiene como objetivo optimizar las preferencias de las familias a la hora de asignarles colegios, así como también buscar la mejor combinación posible para que la mayor cantidad de niños y niñas pueda quedar en el colegio de su preferencia, aun cuando este sistema no garantiza que todos ingresen al establecimiento que es su primera opción, pues es imposible satisfacer la demanda cuando hay más postulantes que vacantes. De esta forma, lo que diferencia el actual sistema respecto del antiguo 
es que ahora todos los postulantes tienen la misma posibilidad de acceder, mientras que antes no todos los niños estaban en igualdad de condiciones para competir por un cupo en un establecimiento educacional.

Es evidente que la selección de los colegios se opone a la libertad de elección de los padres y apoderados, en cambio el NSA representa un mejor aliado al dar un paso importante para ir perfeccionando la libertad de elección en el sistema escolar.

De esta forma — - en teoría—, este nuevo sistema de admisión debería ir disminuyendo la segregación en las escuelas, las que no pueden seguir discriminando por razones económicas o por las notas, en tanto que los colegios con copago tendrán que disminuir el cobro que le hacen a las familias a medida que el Estado aumente el aporte de manera gradual. Es decir, existe la obligación de que el sistema educativo, en su conjunto, propicie espacios educativos heterogéneos.

La implementación de esta ley implica para las "escuelas y sus comunidades, así como para sus equipos docentes y directivos, la modificación significativa de un conjunto de prácticas institucionales profundamente arraigadas en las culturas y tradiciones escolares" (Carrasco y González, 2017, p. 65).

En efecto, en el antiguo sistema de admisión los mecanismos de postulación y admisión estaban descentralizados; además, entre los colegios, el Mineduc y los sostenedores no existía coordinación, de modo que los padres y apoderados debían postular a cada uno de ellos por separado. De hecho, cada establecimiento educacional administraba su propio proceso, bajo la lógica de que los padres y apoderados realizarían una elección informada del establecimiento de acuerdo con sus méritos académicos, principalmente. Sin embargo, este resulta un fenómeno complejo, ya que "ni todos los padres valoran y priorizan los mismos elementos o factores al elegir escuelas para sus hijos, ni todos están en igualdad de condiciones para elegir en función de sus reales preferencias" (Román y Murillo, 2014, p .6), lo cual indica que la elección de escuelas va más allá de la teoría de la acción racional, donde algunas familias tienen una aproximación 
instrumental y realizan cálculos de costo-beneficio al momento de decidir dónde estudiarán sus hijos (Orellana, Caviedes, Bellei y Contreras, 2018), sino que se incluye la ponderación de otros factores tales como los sociodemográficos, los económicos y los culturales.

En un sistema de cuasimercado educativo, la libre elección de una escuela es una condición básica en la que idealmente las familias debiesen tener conocimiento de las diferentes ofertas que presenta el sistema escolar y usar dicha información para elegir las "mejores" escuelas para sus hijos, obligando a las "malas" a perfeccionarse. Esta lógica ha generado una dinámica de oferta y demanda, limitada en gran medida por la capacidad de pago de las familias, provocando un aumento de la cobertura de las escuelas particulares subvencionadas, y una disminución sistemática y crítica de la matrícula en los establecimientos municipales (Valenzuela et al., 2010), aunque con efectos positivos en el sector particular subvencionado, el cual ha incrementado sustancialmente el número de alumnos en todos sus niveles escolares.

Es así como en el contexto del NSA parece relevante comprender cuál es la lógica que motiva a los apoderados al momento de optar por un establecimiento educativo. Al respecto, la clave podría estar dada en el concepto que tienen las familias en cuanto a "una buena o mala escuela". Frente a esto surge otra variable importante de considerar: la influencia que parecieran ejercer los resultados de evaluaciones estandarizadas como el Sistema Nacional de Evaluación de Resultados de Aprendizaje, Simce, -y su correspondiente publicación de resultados en formato ranking - sobre la percepción de calidad de los padres, así como también la determinación de en qué medida estos resultados se constituyen en un referente para la selección del establecimiento educativo.

Al revisar estudios en torno a la elección en el sistema escolar chileno existe muy poca evidencia acerca de las prácticas y racionalidades que las familias despliegan al elegir escuela para sus hijos. Por ejemplo, Carrasco y San Martín (2012) no consideran que la efectividad escolar sea un factor de gran relevancia para la elección de escuelas por parte de las familias; en tanto que Córdoba (2014) 
indica en sus hallazgos que las familias más vulnerables operarían guiadas por consideraciones prácticas como la gratuidad y cercanía, o bien, elementos tradicionales como el conocimiento previo de la escuela y la protección social. En esta misma línea, pero focalizados en los sectores medios, los resultados señalan que estos tienden a distanciarse y diferenciarse de los más pobres — que se asocian a las escuelas municipales-, transformándose en una elección escolar de base clasista (Canales et al., 2016). Dichas prácticas son, precisamente, las que la Ley de Inclusión Escolar pretende eliminar, manifestándose en una serie de derechos estudiantiles respecto de su admisión y permanencia en estos establecimientos educacionales (Ley 20.845, artículo 12, 2015). En este sentido, la eliminación de la selección busca mejorar la calidad del sistema educativo y construir una sociedad más integrada y cohesionada (Mensaje No 131/362, 2014). Con ello, se piensa en establecimientos educacionales con espacios inclusivos donde conviven estudiantes con diferente capital cultural, económico y social. Al respecto, solo el 22,8\% de los estudiantes que acude a colegios socioeconómicamente heterogéneos supera a sus pares de la misma condición social que estudian en establecimientos segregados desaventajados, siendo beneficiados en términos de rendimiento académico en pruebas nacionales estandarizadas de matemática y lenguaje (Ortiz, 2015).

\section{Metodología}

La presente investigación tiene carácter exploratorio, dado que indaga en torno a cómo se comportó la matrícula con el NSA que se implementó como plan piloto en la Región de Magallanes para el proceso 2017. En este contexto, el foco de estudio analiza el mecanismo recientemente aplicado donde los apoderados encuentran información de cada uno de los establecimientos y deben poner sus postulaciones en orden de preferencia.

Este análisis permite identificar datos empíricos y aspectos fundamentales que den una visión general, de tipo aproximativo, respecto del comportamiento de un sistema que busca, primordialmente, evitar que los establecimientos seleccionen estudiantes mediante mecanismos como entrevistas o aplicación de 
pruebas. Para ello, el NSA pondera, en primer término, las preferencias de los apoderados, pero en caso de que hubiera más demanda que vacantes (cuestión que ocurrió en el 28\% de los establecimientos de la región), se prioriza a estudiantes que ya cuenten con hermanos o apoderados trabajando en el establecimiento; a estudiantes que ya estén estudiado ahí; luego se garantiza una cuota de estudiantes prioritarios (vulnerables); y finalmente, en caso de haber más postulaciones que cupos, se aplica una lógica de aleatoriedad. Si un estudiante no encuentra cupo en los establecimientos a los que postula, debe ser redirigido al establecimiento más cercano a su domicilio que cuente con disponibilidad.

Dadas las características de este nuevo sistema, en la investigación se optó por una metodología cuantitativa exploratoriacorrelacional ya que, junto con ser utilizada en diferentes ámbitos desde estudios de opinión hasta diagnósticos para establecer políticas educativas-, permite conocer la distribución de ciertas variables de interés en una población determinada (Hueso y Cascanti, 2012), a través de una medición estandarizada y numérica, características propias de las ciencias exactas (Hernández, Baptista y Fernández, 2003). Además, la metodología es reduccionista y utiliza el análisis estadístico para contestar preguntas de investigación y probar hipótesis previamente establecidas.

Metodológicamente, se analizaron las postulaciones realizadas por los apoderados que utilizaron el nuevo sistema para sondear los resultados de dicho proceso, cuyos detalles se obtuvieron a partir de las bases de datos del Mineduc, facilitadas al Observatorio de Políticas Educativas y Prácticas Pedagógicas de la Universidad de Magallanes, UMAG.

A través de una revisión de las fuentes secundarias, se analizó la conducta de postulación de los apoderados, tomando como unidad de análisis los establecimientos educacionales que recibían las solicitudes. De esa manera, a través de las postulaciones y preferencias que marcaban los establecimientos, se pudo analizar cuáles son los tipos de establecimientos con mayor y menor demanda. El foco principal fue diferenciar, además de lo anterior, las postulaciones de 
los apoderados de estudiantes prioritarios respecto de los apoderados de estudiantes no prioritarios.

Como criterios de exclusión, se eliminó del análisis a los establecimientos con menos de 20 vacantes, principalmente con el objetivo de que las instituciones más pequeñas, sobre todo las que están ubicadas en entornos con baja densidad poblacional, no alteraran los resultados de preferencias con bajo o alto número de cupos, por tratarse de la única oferta, o bien, por existir una baja población en edad escolar. De esta manera, solo se consideraron establecimientos de las comunas de Punta Arenas, Puerto Natales y Porvenir.

Así mismo, se realizó un análisis descriptivo consistente, en primer lugar, en tablas de frecuencia y tablas de contingencia bivariadas, en donde se buscó caracterizar el fenómeno de estudio en su línea base, particularmente la condición de vulnerabilidad en cada establecimiento y la distribución de matrícula de estudiantes vulnerables que se presentaron durante el año 2016, para luego compararla con la distribución de estudiantes vulnerables del año 2017. Para esta segunda parte, se ocuparon coeficientes de correlación parcial en donde se buscó medir el vínculo existente entre la cantidad y la proporción de estudiantes vulnerables que tenía cada establecimiento en el año 2016, en relación con los ingresos 2017. Los coeficientes de correlación se controlaron por "matrícula" y por "vacantes", según correspondiera, para eliminar el sesgo de que el tamaño del establecimiento pudiera afectar el análisis, para lo cual se utilizó el software SPSS 21.

\section{Resultados}

Como primer elemento para caracterizar el proceso de admisión se revisó la situación contextual del año anterior (2016), para mostrar cómo se comportaba la distribución de la matrícula en el sistema municipal y particular subvencionado. 
Tabla 1

Distribución de establecimientos según dependencia y copago en Región de Magallanes

\begin{tabular}{l|l|l|l}
\hline \multicolumn{2}{c|}{$N^{o}$ Establecimientos } & \multicolumn{2}{c}{$N^{o}$ Establecimientos } \\
\hline Sin copago & 25 & Municipal & 22 \\
\hline Con copago & 13 & Particular subvencionado & 16 \\
\hline Total & 38 & Total & 38 \\
\hline
\end{tabular}

Fuente: Elaboración propia.

Tabla 2

Situación 2016. Matrícula por dependencia

\begin{tabular}{lcccc}
\hline & \multicolumn{4}{c}{ Dependencia } \\
\hline & $\begin{array}{c}\text { Matrícula } \\
\text { promedio por } \\
\text { establecimiento }\end{array}$ & Total & $\begin{array}{c}\text { Particular subvencionado } \\
\text { promedio por } \\
\text { establecimiento }\end{array}$ & Total \\
\hline Matrícula prioritarios 2016 & 231 & 5.543 & 153 & 2.452 \\
\hline Matrícula 2016 & 413 & 9.902 & 577 & 9.233 \\
\hline Total & & 15.445 & & 11.685 \\
\hline
\end{tabular}

Fuente: Elaboración propia.

Durante el año 2016, en la matrícula de los establecimientos de la Región de Magallanes se contabilizaban 19.135 estudiantes en los subsistemas municipal y particular subvencionado. De esos, 7.995 estaban considerados "prioritarios", es decir, presentaban alguna condición de vulnerabilidad. Así, sobre la base de la Tabla 2 se puede apreciar que:

- El 69,3\% de esos estudiantes prioritarios estudiaba en algún establecimiento municipal.

- Considerando el total de estudiantes del sistema particular subvencionado, el 21\% de la matrícula de esos establecimientos provenía de estudiantes prioritarios, cifra que asciende al 35\% para establecimientos municipales.

Relacionado con ello, la Junta Nacional de Auxilio Escolar y Becas, Junaeb, otorga un puntaje denominado Índice de Vulnerabilidad (IVE), el cual pondera una serie de variables para finalmente adjudicar un porcentaje para cada establecimiento, el cual 
indicaría la proporción de la matrícula que se encuentra en condición de vulnerabilidad. La manera en la que se distribuye ese porcentaje, considerando la dependencia de los establecimientos educacionales, queda expresada en la Figura 1:

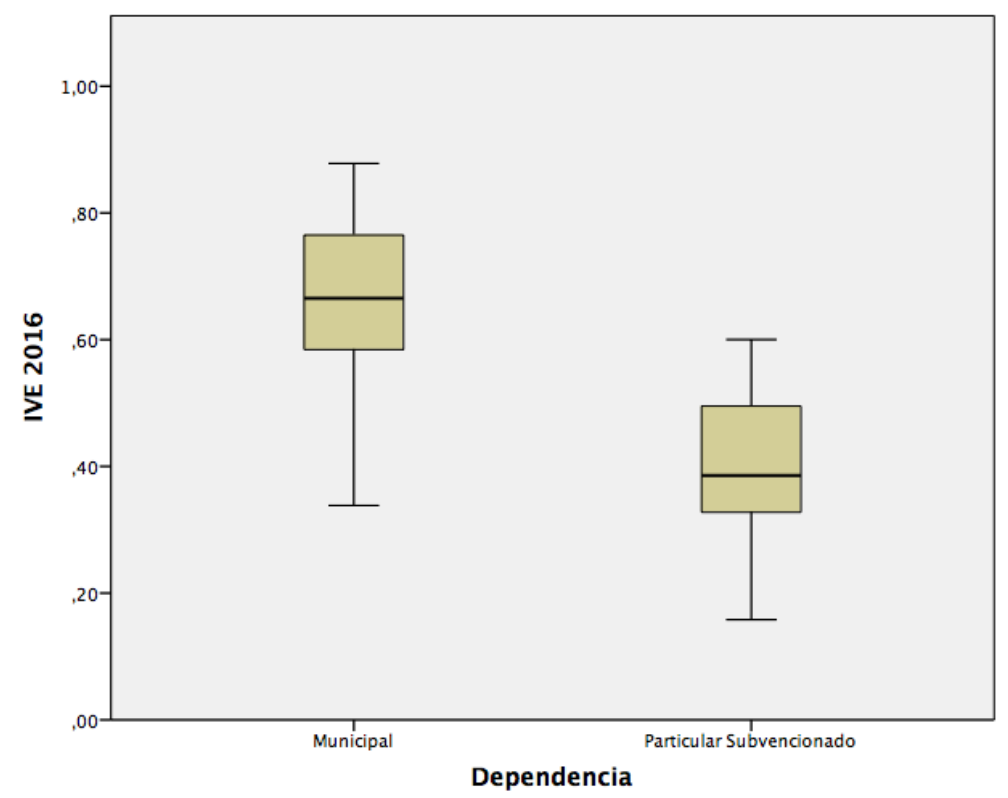

Figura 1. Diagrama de caja. Distribución del IVE 2016 por dependencia.

Fuente: Elaboración propia.

Tal como se puede apreciar, la media del IVE 2016 para establecimientos municipales $(61,1 \%)$ está levemente por encima del puntaje máximo de un establecimiento particular subvencionado (61\%), los que poseen al mismo tiempo a más de la mitad de los establecimientos por debajo de un 40\% de IVE (mediana: 36,7\%).

Del mismo modo, como está ampliamente respaldado en la literatura, la condición socioeconómica de los estudiantes predice, en buena parte, su rendimiento académico; por tanto, la proporción de estudiantes vulnerables que posea un establecimiento será una variable de peso para comprender los resultados de la institución en las pruebas estandarizadas. 
Otros datos indican que hubo 3.664 postulaciones a 4.867 cupos, de las cuales un 64\% quedó en el establecimiento de su primera preferencia, indicador que aumenta a $77 \%$ para las primeras tres preferencias. Esta diferencia entre preferencias y cupos ofertados demuestra que las postulaciones estuvieron concentradas en algunos establecimientos, por tanto, el problema central no radica en la cobertura del sistema, sino en la demanda que existe sobre determinados centros educativos.

Tabla 3

Distribución de las preferencias de los estudiantes en establecimientos sobre la base de su dependencia y copago

\begin{tabular}{lccccc}
\hline & Municipal & $\%$ & $\begin{array}{c}\text { Particular } \\
\text { subvencionado }\end{array}$ & $\%$ & Total \\
\hline $\begin{array}{l}\text { Primeras preferencias } \\
\text { estudiantes prioritarios }\end{array}$ & 314 & $64,7 \%$ & 171 & $35,3 \%$ & 485 \\
\hline $\begin{array}{l}\text { Primeras preferencias } \\
\text { estudiantes no prioritarios }\end{array}$ & 752 & $45,9 \%$ & 887 & $54,1 \%$ & 1.639 \\
\hline \begin{tabular}{l} 
Total primeras preferencias \\
\hline
\end{tabular} & 1.066 & $50,2 \%$ & 1.058 & $49,8 \%$ & 2.124 \\
\hline $\begin{array}{l}\text { Primeras preferencias } \\
\text { estudiantes prioritarios }\end{array}$ & 387 & $79,8 \%$ & 98 & $20,2 \%$ & 485 \\
\hline $\begin{array}{l}\text { Primeras preferencias } \\
\text { estudiantes no prioritarios }\end{array}$ & 890 & $54,3 \%$ & 749 & $45,7 \%$ & 1.639 \\
\hline \begin{tabular}{l} 
Total primeras preferencias \\
\hline
\end{tabular} & 1.277 & $60,1 \%$ & 847 & $39,9 \%$ & 2.124 \\
\hline
\end{tabular}

Fuente: Elaboración propia.

Sin embargo, la cuestión más relevante para analizar el mecanismo es saber si este reordena la matrícula de forma más aleatoria, pudiendo distribuir de manera más equitativa a los estudiantes vulnerables. Sin embargo, al explorar los datos disponibles al respecto, estos estarían indicando que la situación no ha mejorado. En efecto, la distribución de estudiantes prioritarios (o vulnerables) por establecimiento en 2017 sería similar a la del año anterior. Esto ocurriría, principalmente porque el copago (arancel o mensualidad) sigue existiendo, por tanto, las familias de menos ingresos tienen "menos libertad" para escoger y los colegios subvencionados —a pesar de no hacerlo directamente-, continúan seleccionado estudiantes y familias, ya que ponen barreras financieras para el ingreso. 
Cabe mencionar que esta revisión se hace desde una perspectiva modesta, sin considerar datos más complejos que pudieran explicar mejor la distribución de la matrícula al no poder incluir, por ejemplo, la distancia física de los postulantes a los establecimientos. Considerando, además, los factores multivariados que existen para tomar la decisión de escoger escuela, es razonable esperar que el NSA en su fase piloto aún no sea un factor absolutamente decisivo respecto de la distribución de la matrícula.

Para efectos de este sondeo, se revisó en profundidad la forma en que se distribuyeron las preferencias de los apoderados, comparando estudiantes prioritarios con estudiantes no prioritarios, específicamente en las postulaciones para prekínder, kínder y primero básico, las cuales comprendieron un total de 2.154 estudiantes y sus respectivas postulaciones.

Para los casos analizados, las familias de estudiantes prioritarios, en su mayoría, tuvieron como primera opción un establecimiento municipal (65\%), mientras que un 53\% de las familias de estudiantes no prioritarios escogió como primera alternativa un establecimiento particular subvencionado.

El orden de las preferencias de los apoderados comparando familias de estudiantes prioritarios versus no prioritarios entrega una primera pista de cómo funcionó el reciente sistema de admisión. Considerando su orientación y objetivo inicial (que los estudiantes vulnerables tuvieran una concentración más equitativa en los distintos establecimientos producto de la eliminación de los sistemas de selección) ello pareciera no ocurrir si se revisa la asignación final del proceso.

Analizando la Tabla 4, se puede apreciar la relación que existe entre primeras preferencias y asignaciones comparadas por establecimientos con y sin copago y, de esta manera, visibilizar que el factor copago es fundamental para seguir excluyendo las propias primeras preferencias de los estudiantes prioritarios y, por tanto, su asignación final. Lo destacable de este dato es que sin el NSA no se contaría con antecedentes fidedignos de las preferencias de las 
familias en forma simultánea en procesos de postulación, puesto que con el sistema antiguo no era posible ver a cuántos establecimientos postulaba cada estudiante, ni mucho menos la "primera preferencia" en sus prioridades. Es por ello que este tipo de información representa un valioso aporte para ahondar más en el fenómeno de elección de escuela en futuras investigaciones.

Tabla 4

Distribución de las asignaciones finales y las primeras preferencias por establecimientos en función a si tienen o no copago

\begin{tabular}{llrrrrr}
\hline \multicolumn{6}{c}{ Total de postulantes: 2.124 } \\
\hline & \multicolumn{3}{l}{ Estudiantes prioritarios: 485} & \multicolumn{4}{c}{$\begin{array}{c}\text { Estudiantes no prioritarios: } \\
1.639\end{array}$} \\
\hline \multirow{2}{*}{ Primeras preferencias } & Sin copago & 387 & $79,8 \%$ & Sin copago & 890 & $54,3 \%$ \\
\cline { 2 - 7 } & Con copago & 98 & $20,2 \%$ & Con copago & 749 & $45,7 \%$ \\
\hline \multirow{2}{*}{ Asignación final } & Sin copago & & $84 \%$ & Sin copago & $60 \%$ \\
\cline { 2 - 7 } & Con copago & $16 \%$ & Con copago & $40 \%$ \\
\hline
\end{tabular}

Fuente: Elaboración propia.

Dentro de los procesos de postulación y preferencias de los apoderados cabe destacar algunos datos respecto de un establecimiento en particular: el centro municipal más demandado es el Colegio Juan Bautista Contardi (que tiene matrícula de prekínder hasta cuarto año medio). Si se consideran las tres primeras preferencias de todos los postulantes (5.656), el Colegio Juan Bautista Contardi suma 808 (14\%) y ocupa, por lejos, el primer lugar (seguido por el Colegio Charles Darwin, con 465). Además, el establecimiento se caracteriza por: ofrecer solo 71 vacantes; presentar una baja proporción de estudiantes vulnerables (IVE $2016=31,8 \%$ ); contar con una alta reputación por tener buen rendimiento en pruebas estandarizadas (Simce 2016; Lenguaje = 293 y Matemática = 298); y, hasta antes de la implementación de este sistema, ser selectivo en su ingreso ante la alta demanda.

Considerando los datos de la Tabla 4, dentro de la categoría de estudiantes no prioritarios existen 1.639 casos, de los cuales 890 colocaron como primera preferencia un establecimiento sin copago y, de ellos, un 32\% (288 estudiantes) optaron por el Colegio 
Juan Bautista Contardi. Al revisar el dato análogo en estudiantes prioritarios, resulta que solo un $12 \%$ (46 estudiantes) de quienes optaron por establecimientos sin copago (387) eligió dicho colegio como primera opción. ¿Por qué postulan menos familias de estudiantes prioritarios que no prioritarios a un establecimiento municipal de alto rendimiento académico, que no tiene copago ni proceso de selección, gracias al nuevo sistema?

Hay otros datos que ofrecen pistas para "explorar" esto de forma más interactiva. Por ejemplo, ir correlacionando la cantidad de postulaciones que recibió un establecimiento con la cantidad o proporción de estudiantes vulnerables que tenía al año inmediatamente anterior (IVE 2016). La cantidad de preferencias de los apoderados puede verse alterada por los cupos que ofrece el establecimiento (y que por ello más apoderados postulen solo por el tamaño de cupos posibles), por tanto, se establece un cálculo de correlaciones parciales entre el IVE 2016 y las primeras preferencias, controlado por la variable "vacantes".

Tabla 5

Coeficiente de correlación parcial de IVE 2016. Variable de control: vacantes

\begin{tabular}{lccc}
\hline & $\begin{array}{c}\text { Total primeras } \\
\text { preferencias 2017 }\end{array}$ & $\begin{array}{c}\text { Primeras } \\
\text { preferencias } \\
\text { prioritarios 2017 }\end{array}$ & $\begin{array}{c}\text { Primeras } \\
\text { preferencias no } \\
\text { prioritarios 2017 }\end{array}$ \\
\hline Correlación & $-0,534$ & 0,025 & $-0,592$ \\
\hline Significación (bilateral) & 0,001 & 0,889 & $\mathrm{p}<0,001$ \\
\hline Gl & 33 & 33 & 33 \\
\hline
\end{tabular}

Fuente: Elaboración propia.

Lo que se puede apreciar de la Tabla 5 es que la conducta de postulación de los apoderados se correlaciona con la proporción de estudiantes vulnerables que tenga la escuela. Este vínculo es inverso, pues a medida que disminuye el IVE 2016 del establecimiento, aumentan las postulaciones de primera preferencia de los apoderados. Sin embargo, esto no ocurre con el segmento de apoderados de estudiantes prioritarios, cuya cantidad de postulación a los establecimientos no tiene correlato con el IVE 2016, pero sí ocurre para el segmento de estudiantes no prioritarios. 
Al medir la cantidad de estudiantes vulnerables y su distribución en 2016 en los establecimientos y compararla con la distribución de 2017 gracias al nuevo mecanismo, se aprecian las siguientes correlaciones parciales: cuando se mide el vínculo entre la cantidad de estudiantes prioritarios que tenían los establecimientos en 2016 con la cantidad de estudiantes prioritarios que recibieron en 2017, la correlación es bastante alta, e incluso más que la relación entre IVE y primeras preferencias, o sea, además de que los apoderados tienden a reproducir la distribución de la vulnerabilidad del sistema antiguo con sus propias preferencias, es la asignación final del sistema nuevo la que termina consolidándola.

Las correlaciones parciales están diferenciadas, en primer término, por las variables de control por matrícula (una mayor cantidad de matrícula pudiera indicar artificialmente mayor matrícula de estudiantes vulnerables) y, en segundo lugar, por vacantes (puesto que establecimientos con mayor cantidad de vacantes pudieran estar indicando artificialmente una mayor asignación de estudiantes vulnerables); aún así, la correlación es alta y estadísticamente significativa.

Tabla 6

Coeficiente de correlación parcial de matrícula de estudiantes prioritarios 2016

\begin{tabular}{lclc}
\hline \multicolumn{2}{c}{ Variable de control: Matrícula } & \multicolumn{2}{c}{ Variable de control: Vacantes } \\
\hline $\begin{array}{c}\text { Total de prioritarios } \\
\text { asignados 2017 }\end{array}$ & $\begin{array}{c}\text { Total de prioritarios } \\
\text { asignados 2017 }\end{array}$ \\
\hline Correlación & 0,835 & Correlación & 0,78 \\
\hline Significación & $\mathrm{p}<0,001$ & Significación & $\mathrm{p}<0,001$ \\
\hline Gl & 35 & Gl & 33 \\
\hline
\end{tabular}

Fuente: Elaboración propia. 


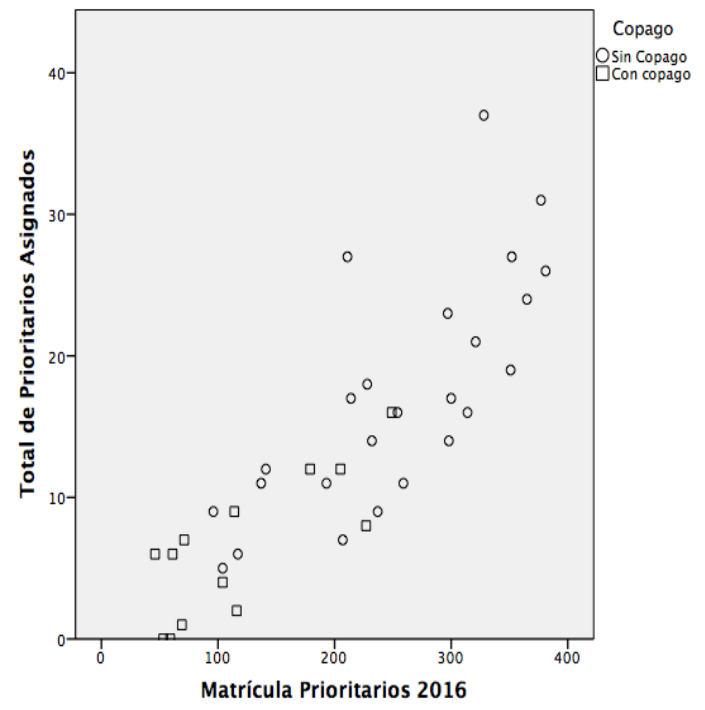

Figura 2. Dispersión de establecimientos considerando matrícula de prioritarios el 2016 y cantidad de estudiantes prioritarios asignados el 2017.

Fuente: Elaboración propia.

Como se puede ver en la Figura 2, existe un vínculo entre matrícula de estudiantes prioritarios 2016 y la cantidad de estudiantes prioritarios que ingresó en 2017. Del mismo modo, se puede apreciar la diferenciación de establecimientos con copago y sin copago: los primeros en el gráfico (círculos), quedan encasillados en la mitad de abajo de la izquierda, recibiendo pocos estudiantes prioritarios; en tanto que los establecimientos sin copago (cuadrados), mientras mayor es su matrícula de estudiantes prioritarios en 2016, más prioritarios reciben en 2017.

Tabla 7

Coeficiente de correlación parcial de IVE 2016

\begin{tabular}{|c|c|c|c|}
\hline \multicolumn{2}{|c|}{ Variable de control: Matrícula } & \multicolumn{2}{|c|}{ Variable de control: Vacantes } \\
\hline & $\begin{array}{l}\% \text { de Prioritarios } \\
\text { asignados } 2017\end{array}$ & & $\begin{array}{l}\text { \% de Prioritarios } \\
\text { asignados } 2017\end{array}$ \\
\hline Correlación & 0,827 & Correlación & 0,852 \\
\hline Significación & $\mathrm{p}<0,001$ & Significación & $\mathrm{p}<0,001$ \\
\hline Gl & 35 & Gl & 33 \\
\hline
\end{tabular}

Fuente: Elaboración propia. 


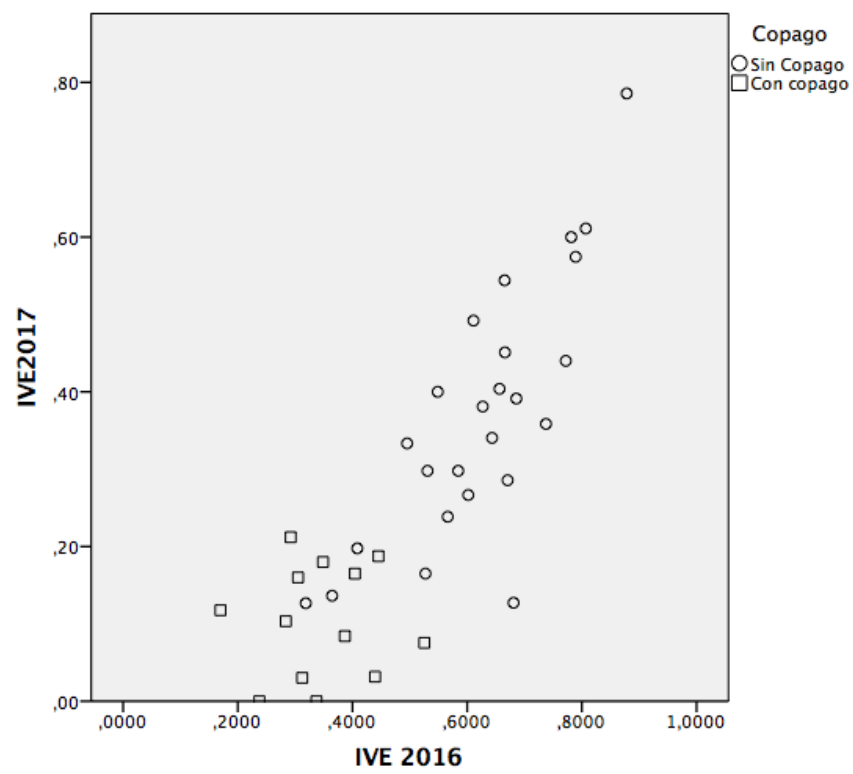

Figura 3. Dispersión de establecimientos considerando IVE 2016 y proporción de estudiantes prioritarios asignados el 2017.

Fuente: Elaboración propia.

Un cálculo similar al anterior se puede hacer comparando los dos porcentajes vinculados con la proporción de estudiantes vulnerables que tiene el establecimiento. Si se considera la medición entre IVE 2016 y la proporción de estudiantes prioritarios en relación con el total de asignados (una especie de IVE 2017, solo considerando estudiantes ingresados con el mecanismo nuevo), nuevamente se tendría una fuerte vinculación que, además, pone en evidencia que las complicaciones de los colegios sin copago-que deben tener una proporción más alta de estudiantes vulnerables-, continúan con el NSA en comparación con el funcionamiento del sistema antiguo.

En este último cálculo de correlación finalmente se evidencia con más claridad que la proporción de estudiantes vulnerables que tenía un establecimiento en 2016 es, casi en su totalidad, predictor de la proporción de estudiantes vulnerables que quedará matriculado en 2017 con el sistema de admisión nuevo. 
Por otro lado, también interesaba a la investigación determinar si los puntajes Simce de los establecimientos correspondientes al año anterior al proceso de selección, tenían algún vínculo con las preferencias de los apoderados. No obstante, los puntajes Simce de los establecimientos están muy correlacionados con los niveles de vulnerabilidad, de modo que al medir la correlación entre los puntajes de la prueba y la cantidad de preferencias de los establecimientos, emergió una correlación directa estadísticamente significativa para la suma de las tres primeras preferencias, para la suma de primeras preferencias y para ambas variables (suma de primeras preferencias y suma de las tres primeras preferencias) de postulación de los estudiantes no prioritarios. De esta manera, las postulaciones de estudiantes prioritarios fueron las únicas sin vínculo con el Simce y pareciera que la conducta de estos apoderados no tendría relación alguna con los puntajes de dicha prueba. Sin embargo, cuando vuelven a aparecer los cálculos de correlación entre preferencias y puntajes Simce —esta vez controlados por el IVE 2016-, las correlaciones significativas desaparecen y deja de haber un vínculo entre el Simce y la cantidad de preferencias que acumulan los establecimientos. 
Tabla 8

Coeficientes de correlación entre puntajes Simce y suma de preferencias recibidas por los establecimientos

Variable de control: IVE 2016

\begin{tabular}{|c|c|c|c|c|c|c|c|}
\hline & & 1 & 2 & 3 & 4 & 5 & 6 \\
\hline \multirow{3}{*}{$\begin{array}{l}\text { Simce Lenguaje } 2016 \\
\text { cuarto año básico }\end{array}$} & Correlación & 0,483 & 0,011 & 0,531 & 0,507 & 0,036 & 0,552 \\
\hline & Significación & 0,003 & 0,949 & 0,001 & 0,002 & 0,838 & 0,001 \\
\hline & Gl & 33 & 33 & 33 & 33 & 33 & 33 \\
\hline \multirow{4}{*}{$\begin{array}{l}\text { Simce Matemática } \\
2016 \text { cuarto año } \\
\text { básico }\end{array}$} & Correlación & 0,499 & 0,007 & 0,549 & 0,548 & 0,063 & 0,592 \\
\hline & Significación & 0,002 & 0,967 & 0,001 & 0,001 & 0,718 & 0 \\
\hline & Gl & 33 & 33 & 33 & 33 & 33 & 33 \\
\hline & & 1 & 2 & 3 & 4 & 5 & 6 \\
\hline \multirow{3}{*}{$\begin{array}{l}\text { Simce Lenguaje } 2016 \\
\text { cuarto año básico }\end{array}$} & Correlación & 0,214 & 0,031 & 0,243 & 0,255 & 0,065 & 0,28 \\
\hline & Significación & 0,217 & 0,861 & 0,159 & 0,14 & 0,709 & 0,103 \\
\hline & $\mathrm{Gl}$ & 33 & 33 & 33 & 33 & 33 & 33 \\
\hline \multirow{3}{*}{$\begin{array}{l}\text { Simce Matemática } \\
2016 \text { cuarto año } \\
\text { básico }\end{array}$} & Correlación & 0,227 & 0,038 & 0,256 & 0,304 & 0,108 & 0,329 \\
\hline & Significación & 0,19 & 0,829 & 0,138 & 0,075 & 0,537 & 0,054 \\
\hline & Gl & 33 & 33 & 33 & 33 & 33 & 33 \\
\hline
\end{tabular}

Nota: (1) Suma tres primeras preferencias; (2) Suma tres primeras preferencias estudiantes de prioritarios; (3) Suma tres primeras preferencias estudiantes no prioritarios; (4) Primeras preferencias total; (5) Primeras preferencias de estudiantes prioritarios; (6) Primeras preferencias de estudiantes no prioritarios.

Fuente: Elaboración propia

\section{Conclusiones}

Se espera que la implementación de la Ley de Inclusión permita la efectiva elección de escuelas por parte de las familias chilenas. A la luz de los datos, el reciente sistema de selección es bastante eficiente en generar un nuevo entorno en donde los establecimientos ahora tienen bajísima capacidad para manipular la demanda estudiantil a su favor y quedarse con estudiantes con más estímulos académicos o de otro tipo. Sin embargo, el problema persiste respecto de la distribución de estudiantes vulnerables en el sistema, pues esta es muy similar a la del sistema antiguo, lo cual se debe en gran medida a que el nuevo mecanismo recién está siendo implementado.

Así mismo, reducir la segregación social entre las escuelas y generar espacios educativos socialmente más heterogéneos "es el 
objetivo más desafiante, puesto que se enfrenta a patrones culturales históricamente constituidos que luchan por mantener las posiciones sociales" (Queupil y Durán del Fierro, 2018, p. 124). Lo que sí está claro por ahora es que el NSA protege el derecho de los padres y apoderados a elegir el establecimiento para sus hijos y este proceso no está limitado por decisiones arbitrarias de los establecimientos educacionales.

Sobre la base de esto, las conclusiones se dividen en dos dimensiones: el efecto del copago y la priorización de un determinado tipo de establecimiento, que a su vez genera una brecha peculiar en torno a la demanda versus los cupos ofertados.

En cuanto a la primera dimensión —el efecto del copago—si bien el nuevo mecanismo de admisión busca que los establecimientos no tengan la capacidad para filtrar a sus estudiantes por razones económicas, esto sigue ocurriendo en los establecimientos particulares subvencionados a causa del cobro de aranceles. La barrera financiera del cobro mensual sigue siendo eficiente para marginar a estudiantes vulnerables y, a la inversa, para capturar apoderados con capacidad y voluntad de pago de la educación de sus hijos. El nuevo sistema de selección, por tanto, —al menos en el segmento de establecimientos con copago-, no genera un reordenamiento de la matrícula a favor de optimizar gradualmente la recomposición social de las escuelas, que en su mayoría concentran a estudiantes con atributos homogéneos, o bien, en traspasar la diferencia socioeconómica de la matrícula "entre" establecimientos hacia "dentro" de los establecimientos: la proporción de alumnos prioritarios - que limita a un $15 \%$ de alumnos vulnerables que un establecimiento puede priorizar - es un problema anterior a la implementación, lo que implica mantener las desigualdades de origen de los estudiantes al interior de las escuelas.

Vale la pena notar, además, que el efecto del copago (que los establecimientos segreguen socioeconómicamente en función al segmento de ingresos familiares), no solo se ejerce en establecimientos particulares subvencionados con copago, sino que tiene un efecto interactivo con todos los demás. Por ejemplo, si se toma solamente 
a los establecimientos sin copago, sigue estando correlacionada la demanda de los apoderados con la segregación (es decir, existen más preferencias en la medida en que baje la cantidad y proporción de estudiantes vulnerables), aunque no en todos esos establecimientos exista arancel. Por tal motivo, es necesario profundizar los estudios en esta área, ya que la "elección de escuela es un proceso que debe ser analizado tomando en cuenta también sus relaciones con la estructura social, la geografía urbana, las características sociodemográficas de la población y la cultura" (Orellana et al., 2018, p. 13).

El ejemplo citado en el análisis de datos relacionado con el Colegio Juan Bautista Contardi pone en evidencia este punto: siendo un establecimiento de alta demanda y sin copago, comparativamente, postulan menos familias vulnerables que las no vulnerables. De esta manera, acompañando al efecto del copago (no por sí solo), existen otros elementos que aportan al proceso de segregación a la hora de escoger escuela para que, finalmente, la distribución de los estudiantes vulnerables - al parecer - termine siendo igual que con el sistema antiguo. En otras palabras, el nuevo mecanismo de selección no estaría actuando sobre los elementos clave que determinan la elección de establecimiento por parte de los apoderados para poder revertir el proceso de segregación. Esto a lo mejor se debe a que la mayoría de padres y apoderados construye representaciones sociales diferenciadas para cada tipo de establecimiento, producto de la información de que disponen y de su concepción acerca de cómo debe ser la educación que reciben sus hijos (Rogero-García y Andrés-Candelas, 2016). Esto lleva la necesidad de buscar otras herramientas que ayuden a la ley y al sistema a lograr su objetivo de ir construyendo colegios más heterogéneos.

Ahora bien, en cuanto a la segunda dimensión de las conclusiones, esto es, la priorización en la selección del establecimiento a postular y, a partir de la revisión de datos que se desprenden de la primera aplicación del mecanismo de selección, cabe también plantearse algunas interrogantes. Por ejemplo, gracias al NSA se pueden ver con nitidez y transparencia algunos elementos que no eran visibles con el sistema antiguo, entre los que se cuenta la real demanda que tienen buena parte de los establecimientos 
públicos (municipales) y la cantidad de rechazos cuando las familias son asignadas a estos. Esto puede deberse a que se trata de padres y apoderados conscientes de que el origen socioeconómico y cultural del grupo de pares influye significativamente en los resultados académicos individuales (Salinas Jiménez y Santín González, 2012).

A partir de esta puesta en evidencia, recobra cierto sentido la idea de que un sistema de selección centralizado como este debiera ser un punto de llegada más que un punto de partida, que era la crítica que se desprendió de algunos apoderados tras comprender cómo funcionaba el nuevo mecanismo: en alguna dimensión el sistema considera equivalentes y con igual cualidad a establecimientos diferentes, con distinta infraestructura, composición del estudiantado, teniendo copago o no, siendo municipal o subvencionado, pero esto no es así en la realidad, con lo cual persisten incentivos objetivos para no postular a un determinado colegio, o bien, para rechazar la asignación, principalmente cuando se trata de los establecimientos públicos. En ese caso, y antes de implementar un nuevo sistema de selección, cabe preguntarse si acaso no sería más positivo primero potenciar, invertir y mejorar la educación pública y luego colocarla en un sistema en donde opere en algún sentido de aleatoriedad.

Con esto en mente resultaría muy pertinente que durante los primeros años de implementación del NSA existiera una evaluación continua del mismo, ya que ello permitiría detectar las falencias de forma temprana e introducir mejoras rápidamente, distinguiendo de paso los problemas de diseño de la ley y los que tienen relación directa con la implementación de ella a través del NSA.

\section{Referencias}

Bellei, C. (2009). The private-public school controversy: The case of Chile. En P. Peterson \& R. Chakrabarti (Eds.), School choice international (pp. 165-192). Cambridge, MA: MIT Press.

Canales, M., Bellei, C., y Orellana, V. (2016). ¿Por qué elegir una escuela privada subvencionada? Sectores medios emergentes y elección de escuela en un sistema de mercado. Estudios Pedagógicos (Valdivia), 42(3), 89-109. https://doi.org/10.4067/s0718-07052016000400005 
Carrasco, A., Bogolasky, F., Flores, C., Gutiérrez, G., y San Martín, E. (2014). Selección de estudiantes y desigualdad educacional en Chile: ¿Qué tan coactiva es la regulación que la prohíbe? Santiago de Chile: Mineduc.

Carrasco, A. y González, P. (2017). Liderazgo para la inclusión y para la justicia social: el desafío del liderazgo directivo ante la implementación de la Ley de Inclusión Escolar de Chile. Educación y Ciudad, 33, 63-74.

Carrasco, A. y San Martín, E. (2012). Voucher system and school effectiveness: Reassessing school performance difference and parental choice decision-making. Estudios de Economía. 39(2): 123-141.

Contreras, D., Bustos, S., y Sepúlveda, P. (2010). When schools are the ones that choose: The effect of screening in Chile. Social Science Quarterly, 91(5), 1349-1368. https://doi.org/10.1111/j.1540-6237.2010.00735.x

Córdoba, Claudia. (2014). La elección de escuela en sectores pobres: Resultados de un estudiocualitativo. Psicoperspectivas, 13(1), 56-67. https://dx.doi.org/10.5027/psicoperspectivas-Vol13-Issuelfulltext-301

Elacqua, G. (2012). The impact of school choice and public policy on segregation: Evidence from Chile. International Journal of Educational Development, 32(3), 444-453.

https://doi.org/10.1016/j.ijedudev.2011.08.003

Hernández, V., Baptista, P., y Fernández, C. (2003). Metodología de la investigación. México D.F.: Mc Graw Hill.

Hueso, A. y Cascanti, M. (2012). Metodología y técnicas cuantitativas de investigación. Cuadernos docentes en procesos de desarrollo Número 1. Valencia: Universitat Politécnica de València.

Ley 20.370. Ley General de Educación del Ministerio de Educación. Diario Oficial de la República de Chile, 12 de septiembre de 2009. Recuperado de http://www.leychile.cl/Navegar?idNorma=1006043

Mensaje No 131/362. (2014). Cámara de Diputados de la República de Chile. Santiago de Chile.

Ley 20.845 De Inclusión Escolar que regula la admisión de los y las estudiantes, elimina el financiamiento compartido y prohíbe el lucro en establecimientos educacionales que reciben aportes del Estado del Ministerio de Educación. Diario Oficial de la República de Chile, 8 de junio de 2015. Recuperado de https://www.leychile.cl/ Navegar?idNorma $=1078172$

Orellana, V., Caviedes, S., Bellei, C., y Contreras, M. (2018). La elección de escuela como fenómeno sociológico. Una revisión de literatura. Revista Brasileira de Educação, 23, 1-19.

http://dx.doi.org/10.1590/S1413-24782018230007 
Ortiz, I. (2015). Escuelas inclusivas en el contexto de segregación social del sistema escolar chileno. Calidad en la Educación, 42, 93-122. https://doi.org/10.4067/s0718-45652015000100004

Queupil, J. P. y Durán del Fierro, F. (2018). El principio de inclusión: similitudes y diferencias en la educación escolar y superior en Chile. Revista Latinoamericana de Educación Inclusiva, 12(1), 111-128. https://dx.doi.org/10.4067/S0718-73782018000100111

Rojas, M. T., Falabella, A., y Alarcón, P. (2016). Inclusión social en las escuelas: estudio de prácticas pedagógicas inclusivas y proyecciones para enfrentar un escenario sin copago y selección escolar (Informe final FONIDE 911429). Santiago de Chile: Mineduc.

Román, M. y Murillo, F. J. (2014). Uso de los resultados de las evaluaciones estandarizadas como criterio de elección y selección de escuelas. Revista Iberoamericana de Evaluación Educativa, 7(1), 5-7. Recuperado de https://dialnet.unirioja.es/descarga/articulo/4704188.pdf

Rogero-García, J. y Andrés-Candelas, M. (2016). Representaciones sociales de los padres y madres sobre la educación pública y privada en España. Revista de la Asociación de Sociología de la Educación, 9(1), 46-58.

Salinas Jiménez, J. y Santín González, D. (2012). Selección escolar y efectos de la inmigración sobre los resultados académicos españoles en PISA 2006. Revista de Educación, 358, 382-405. Recuperado de http://www. revistaeducacion.educacion.es/re358/re358_18.pdf

Valenzuela, J., Bellei, C., y Ríos, D. (2010). Segregación escolar en Chile. En S. Martinic y G. Elacqua (Coords.), ¿Fin de ciclo? Cambios en la gobernanza del sistema educativo (pp. 209-229). Santiago de Chile: Unesco. Recuperado de http://unesdoc.unesco.org/ images/0019/001905/190544S.pdf

Valenzuela, J., Bellei, C., \& Ríos, D. (2014). Socioeconomic school segregation in a market-oriented educational system. The case of Chile. Journal of Education Policy, 29(2), 217-241.

https://doi.org/10.1080/02680939.2013.806995

Recibido: 02/01/2018

Aceptado: 24/07/2018 